\title{
Geometric Expansion of the Boundary Free Energy of a Dilute Gas
}

\section{Pierre Collet and François Dunlop}

Centre de Physique Théorique de l'Ecole Polytechnique, Plateau de Palaiseau, F-91128 Palaiseau Cedex, France

\begin{abstract}
We consider a dilute classical gas in a volume $\varepsilon^{-1} \Lambda$ which tends to $\mathbb{R}^{d}$ by dilation as $\varepsilon \rightarrow 0$. We prove that the pressure $p\left(\varepsilon^{-1} \Lambda\right)$ is $C^{q}$ in $\varepsilon$ at $\varepsilon=0$ (thermodynamic limit), for any $q \in \mathbb{N}$, provided the boundary $\partial \Lambda$ is $C^{q}$ and provided the Ursell functions $u_{n}\left(x_{1}, \ldots, x_{n}\right)$ admit moments of degree $q$ and have "nice" derivatives.
\end{abstract}

\section{Introduction}

In a recent paper [1], Pogosian derives the asymptotic expansion of the pressure $p\left(\varepsilon^{-1} \Lambda\right)$ in the thermodynamic limit $\varepsilon \rightarrow 0$, up to order $d$ in $\varepsilon$,

$$
p\left(\varepsilon^{-1} \Lambda\right)=a_{0}(\Lambda)+a_{1}(\Lambda) \varepsilon+\cdots+a_{d-1}(\Lambda) \varepsilon^{d-1}+a_{d}(\Lambda) \varepsilon^{d}+r_{d}(\varepsilon, \Lambda) \varepsilon^{d}
$$

for a dilute gas in $\Lambda \subset \mathbb{Z}^{d}$ or $\Lambda \subset \mathbb{R}^{d}$. The remainder satisfies

$$
\left|r_{d}(\varepsilon, \Lambda)\right| \leqq \begin{cases}0\left(\log \varepsilon^{-1}\right) & \text { in general } \\ 0(1) & d=2 \\ 0(\varepsilon) & \text { if } \Lambda \subset \mathbb{Z}^{d} \text { or } \Lambda \text { polyhedron in } \mathbb{R}^{d} .\end{cases}
$$

The hypotheses on $\partial \Lambda$ are the natural ones, the hypotheses on the interaction potential are rather complicated and are not optimal. The proof is based on the Mayer expansion and extensive use of Taylor expansions.

The present paper extends the above results and simplifies the proofs, for volumes $\varepsilon^{-1} \Lambda \subset \mathbb{R}^{d}$ with $\partial \Lambda$ smooth. We prove the absence of logarithms (as conjectured by Pogosian), and extend the expansion to all orders. The order $d$ (dimension of space) has nothing special to it when the interaction is smooth, which we assume, as Pogosian does in his proof if we understand it correctly. It is clear however that strong singularities in the interaction potential would show up in the expansion at some order in $\varepsilon$ depending on the dimension. We do not know whether a jump discontinuity in the Ursell functions (e.g. square hard core potential) would spoil the expansion at all. 
Our hypotheses on the long range behaviour of the potential are the minimal hyjpotheses for the absolute convergence of the sums and integrals which define the coefficients of the expansion in the limit $\varepsilon=0$.

It is well known that $a_{0}(\Lambda)$ and $a_{1}(\Lambda)$ are proportional respectively to $|\Lambda|$ and $|\partial \Lambda|$. Pogosian has shown (for $\Lambda$ smooth):

$$
a_{2}(\Lambda) \approx \int_{\partial \Lambda} H(x) d x
$$

where $H(x)$ is the mean curvature at $x \in \partial \Lambda$. We remark that the two point Ursell function does not contribute to $a_{2}(\Lambda)$ :

$$
\varepsilon^{d} \int_{\left(\varepsilon^{-1} \Lambda\right)^{2}} d x d y U_{2}(x-y)=b_{0}|\Lambda|+\varepsilon b_{1}|\partial \Lambda|+\varepsilon^{3} b_{3}\left\langle R^{-2}\right\rangle_{\partial \Lambda}+O\left(\varepsilon^{4}\right),
$$

where $R^{-1}$ denotes the normal curvature in a plane normal to $\partial \Lambda$ at $x \in \partial \Lambda$, and the average is taken over the orientation of the normal plane and over $x \in \partial \Lambda$. This explicit formula assumes rotation invariance, and is valid only for the two point function. General results and formulae for the $n$-point integral are given in Theorem 2. In general $a_{j}(\Lambda)$ will be a finite sum (not just one term as above), where each term is factorized into a potential dependent factor times a volume dependent factor.

Corollary 1 combines Theorem 2 with the Mayer expansion for the pressure, at small enough activity. Corollary 2 extends the results to the whole analyticity region in the activity, assuming bounds on the truncated correlation functions; it also gives recursion formulae for the expansion in $\varepsilon$ of the average number of particles in volume $\varepsilon^{-1} \Lambda$.

Theorem 1 is the hard core of the paper, which we now explain in words. (The reader interested in final results might now go to Theorem 2 and corollaries).

The idea of our derivation is to write a Taylor expansion around $\varepsilon=0$ of $p\left(\varepsilon^{-1} \Lambda\right)$. This will involve of course computing derivatives of that quantity. These derivatives will be expressed in terms of various integrals containing derivatives of the Mayer functions. Explicit expressions for these integrals will be determined by recursion over the order of derivation in $\varepsilon$. Due to the presence of $\varepsilon^{-1}$ in the function $p\left(\varepsilon^{-1} \Lambda\right)$, derivations in $\varepsilon$ will produce terms which are apparently singular in $\varepsilon=0$. It turns out that under our regularity hypothesis these singularities cancel. This compensation is performed explicitly by using integration by parts over the space variables. Therefore a typical term in the Taylor expansion of $p\left(\varepsilon^{-1} \Lambda\right)$ will be a sum of integrals with some variables being integrated over the domain $\Lambda$, and the remaining variables over the boundary $\partial \Lambda$ of $\Lambda$. One can observe that two scales are present in the problem: the fixed scale of $\Lambda$, and the varying (large) scale $\varepsilon^{-1}$. This implies that the integrands can be regarded as functions of the space variables $x$ on the one hand and of $y=\varepsilon^{-1} x$ on the other hand.

Lemma 2 deals with the derivation with respect to $\varepsilon$ of such integrals. Lemma 3 is an identity from differential geometry which is used to exhibit cancellations of singular terms in the integration by parts (see for example [3] for the method of the moving frame). As explained above, our main result follows by recursive applications of Lemma 2. 


\section{Results}

We first give a local result (Theorem 1 , where the $n$-point function is anchored at $x_{1}=0$ ), then a global result with detailed formulae (Theorem 2), which we apply to the Mayer expansion for the pressure (Corollary 1); Corollary 2 deals with the number of particles, with hypotheses bearing on correlation functions rather than on Ursell functions.

Theorem 1. Let $\Lambda \subset \mathbb{R}^{d}, 0 \in \partial \Lambda, \partial \Lambda$ a $C^{r}$ hypersurface. Given integers $n \geqq p_{0} \geqq 1$, let

$$
u\left(x_{2}, \ldots, x_{n} ; y_{2}, \ldots, y_{n}\right) \in C^{k}\left((\partial \Lambda)^{p_{0}-1} \times \Lambda^{n-p_{0}} \times R^{(n-1) d}\right) .
$$

Let $0 \leqq q \leqq \inf (k, r-1)$ and suppose that all derivatives $D^{\alpha} u$ of total order $0 \leqq|\alpha| \leqq q$ satisfy

$$
{\left(\varepsilon^{-1} \partial \Lambda\right)^{p-1}}_{\left.\int_{\times(\varepsilon}-1 \Lambda\right)^{n-p}} d y_{2} \cdots d y_{n} \prod_{2}^{p}\left|y_{j}\right|^{q_{j}}\left|D^{\alpha} u\left(\varepsilon y_{2}, \ldots, \varepsilon y_{n} ; y_{2}, \ldots, y_{n}\right)\right|<c<\infty
$$

for all $\varepsilon \in\left[0, \varepsilon_{0}\right]$, for all $p$ with $p_{0} \leqq p \leqq n$, and for all $\left\{q_{j}\right\}$ with

$$
q_{j} \geqq 0, \quad \sum_{2}^{p} q_{j} \leqq q+|\alpha|+p-p_{0} .
$$

Suppose moreover that the above integrals are convergent as any $\left|y_{j}\right| \rightarrow+\infty$, uniformly in the other variables (specially $\varepsilon$ ).

Then the function

$$
I_{n, p_{0}}(\varepsilon)=\int_{\left(\varepsilon^{-1} \partial \Lambda\right)^{p_{0}-1}} \int_{\times\left(\varepsilon^{-1} \Lambda\right)^{n-p_{0}}} d y_{2} \ldots d y_{n} u\left(\varepsilon y_{2}, \ldots, \varepsilon y_{n} ; y_{2}, \ldots, y_{n}\right)
$$

is $C^{q}$ at $\varepsilon=0$.

Remark 1. If we assume $\partial \Lambda$ of class $C^{r}$ only near the origin, we can conclude that $I_{n, p_{0}}(\varepsilon)$ is the sum of a $C^{q}$ function and an $0\left(\varepsilon^{q}\right)$ function.

Remark 2. The function $u$ is required to exist on a much wider domain than is necessary to define the integral $I_{n, p_{0}}$. The reason for this is that the domain of integration has a singularity at $\varepsilon=0$.

The next three lemmas will be the basis of the proof of Theorem 1 and also shed some light on its formulation.

Lemma 1. Let $\Lambda \subset \mathbb{R}^{d}, 0 \in \partial \Lambda, \partial \Lambda$ a $C^{r}$ hypersurface, let $n(z)$ be the inner normal to $\partial \Lambda$ at $z$. Then there is a function $Q: \partial \Lambda \times \mathbb{R}^{d} \rightarrow \mathbb{R}$, homogeneous of degree 2 in the second variable and $\mathrm{C}^{-2}$ such that

$$
\varepsilon^{-2} z \cdot n(z)=\left.Q(z, y)\right|_{y=\varepsilon^{-1} z}
$$

Proof. There is an $\varepsilon$ independent neighborhood $V$ of 0 in $\mathbb{R}^{d}$ such that

1) If $z \in \partial \Lambda \cap V$, then $z t \in V \forall t \in[0,1]$.

2) If $x \in V$, the orthogonal projection $P(x)$ from $x$ onto $\partial \Lambda$ is differentiable in $V$, and the vector $x-P(x)$ realizes the shortest distance between $x$ and $\partial \Lambda$.

Let $\chi$ be a $C^{\infty}$ function from $\mathbb{R}^{d}$ to $\mathbb{R}^{+}$such that $\chi \equiv 1$ on some neighborhood of 0 and $\chi \equiv 0$ outside $V$. 
We define a function $Q^{(2)}(z, y)$ by

$$
Q^{(2)}(z, y)=\frac{z \cdot n(z)}{\|z\|^{2}}(1-\chi(z))\|y\|^{2} .
$$

This function is $C^{r-1}$ in $z$ and quadratic in $y$. Moreover, if $z \notin V$,

$$
z \cdot n(z)=\left.Q^{(2)}(z, y)\right|_{y=\varepsilon^{-1}} \cdot
$$

For $z \in \partial \Lambda$ and $t \in[0,1]$, let

$$
g(t)=P(t z) \cdot n(P(t z)) \chi(t z) .
$$

This function is well defined for any $z \in \partial \Lambda$, and any $t \in[0,1]$. From $g(0)=0$, we have

$$
g(1)=\int_{0}^{1} \chi(t z) P(t z) \cdot D n_{P(t z)}\left(D P_{t z}(z)\right) d t+\int_{0}^{1} P(t z) \cdot n(P(t z)) D \chi_{t z}(z) d t
$$

(the term $D P$-drops out).

From $P(0)=0$, we have if $t z \in V$,

$$
P(t z)=\int_{0}^{t} D P_{\tau z}(z) d \tau
$$

We now define a function $Q^{(1)}(z, y)$ by

$$
\begin{aligned}
Q^{(1)}(z, y)= & \int_{0}^{1} d t \chi(t z) \int_{0}^{t} d \tau D P_{\tau z}(y) \cdot D n_{P(t z)}\left(D P_{t z}(y)\right) \\
& +\int_{0}^{1} d t D \chi_{t z}(y) \int_{0}^{t} d \tau D P_{\tau z}(y) \cdot n(P(t z)) .
\end{aligned}
$$

Notice that by the star-shape of $V, Q^{(1)}$ is well defined for any $z$ in $\partial \Lambda$, since $t z \in V$ implies $\tau z \in V$ for any $\tau \in[0, t] . Q^{(1)}$ is obviously $C^{r-2}$ in $z$ and quadratic in $y$. Moreover

$$
g(1)=P(z) \cdot n(P(z))=z \cdot n(z)=Q^{(1)}(z, z) .
$$

The result follows if we set $Q(z, y)=Q^{(1)}(z, y)+Q^{(2)}(z, y)$.

Lemma 2. Formulae for $I_{n, p}(\varepsilon)$ and its first derivative:

$$
\begin{aligned}
& I_{n, p}(\varepsilon)=\varepsilon^{-(n-1) d+p-1} \int_{(\partial \Lambda)^{p-1} \times \Lambda^{n-p}} d x_{2} \ldots d x_{n} u\left(x_{2}, \ldots, x_{n} ; \varepsilon^{-1} x_{2}, \ldots, \varepsilon^{-1} x_{n}\right) \\
& \frac{d}{d \varepsilon} I_{n, p}(\varepsilon)=\varepsilon^{-(n-1) d+p-1} \int_{(\partial \Lambda)^{p-1} \times \Lambda^{n-p}} d x_{2} \cdots d x_{n} v\left(x_{2} \cdots ; \varepsilon^{-1} x_{2} \cdots\right) \\
& +\sum_{j=p+1}^{n} \varepsilon^{-(n-1) d+p} \int_{(\partial \Lambda)^{p}} d x_{2} \cdots d x_{p} d x_{j} \int_{\Lambda^{n-p-1}} d x_{p+1} \cdots d \check{x}_{j} \cdots d x_{n} w_{j}\left(x_{2} \cdots ; \varepsilon^{-1} x_{2} \cdots\right),
\end{aligned}
$$


where

and

$$
\begin{aligned}
v\left(x_{2}, \ldots, x_{n} ; y_{2}, \ldots, y_{n}\right)= & -\sum_{j=2}^{p} Q\left(x_{j}, y_{j}\right)\left(n_{j} \cdot \frac{\partial}{\partial y_{j}} u\right) \\
& +\sum_{j=2}^{p}(d-1) H\left(x_{j}\right)\left(y_{j} \cdot n_{j}\right) u+\sum_{j=2}^{n} y_{j} \cdot \frac{\partial u}{\partial x_{j}}
\end{aligned}
$$

$$
w_{j}\left(x_{2}, \ldots, x_{n} ; y_{2}, \ldots, y_{n}\right)=Q\left(x_{j}, y_{j}\right) u \text {. }
$$

where $Q(x, y)$ is defined in Lemma 1 and $H\left(x_{j}\right)$ is the mean curvature as defined in Lemma 3.

Proof. The formula for $I_{n, p}(\varepsilon)$ is obvious from the definition. We now compute

$$
\begin{aligned}
& \frac{d}{d \varepsilon} I_{n, p}(\varepsilon)=[-(n-1) d+p-1] \varepsilon^{-(n-1) d+p-2} \int \cdots u \cdots \\
& -\sum_{j=2}^{n} \varepsilon^{-(n-1) d+p-3} \int_{(\partial \Lambda)^{p-1} \times \Lambda^{n-p}} d x_{2} \cdots d x_{n} x_{j} \cdot \frac{\partial}{\partial y_{j}} u\left(x_{2}, \ldots, x_{n} ; \varepsilon^{-1} x_{2}, \ldots, \varepsilon^{-1} x_{n}\right) .
\end{aligned}
$$

In the last term, notice that for $2 \leqq j \leqq p, \varepsilon^{-1} x_{j}$ belongs to $\varepsilon^{-1} \partial \Lambda$. However the function $u$ is defined and differentiable in a much larger domain, this is why the above formula is true.

Let us first consider $j \geqq p+1$ in the last term. Integration by parts on $\Lambda$ gives

$$
\begin{aligned}
- & \sum_{j=p+1}^{n} \varepsilon^{-(n-1) d+p-3} \int_{(\partial \Lambda)^{p-1} \times \Lambda^{n-p}} d x_{2} \cdots d x_{n} x_{j} \cdot\left(\frac{\partial}{\partial y_{j}} u\right)\left(x_{2} \cdots x_{n} ; \varepsilon^{-1} x_{2} \cdots \varepsilon^{-1} x_{n}\right) \\
= & (n-p) d \varepsilon^{-(n-1) d+p-2} \int_{(\partial \Lambda)^{p-1} \times \Lambda^{n-p}} d x_{2} \cdots d x_{n} u \\
& +\sum_{j=p+1}^{n} \varepsilon^{-(n-1) d+p-2} \int_{(\partial \Lambda)^{p-1} \times \Lambda^{n-p}} d x_{2} \cdots d x_{n} x_{j} \cdot \frac{\partial}{\partial x_{j}} u \\
& +\sum_{j=p+1}^{n} \varepsilon^{-(n-1) d+p} \int_{(\partial \Lambda)^{p}} d x_{2} \cdots d x_{p} d x_{j} \int_{\Lambda^{n-p-1}} d x_{p+1} \cdots d \check{x}_{j} \cdots d x_{n}\left(\varepsilon^{-2} x_{j} \cdot n_{j}\right) u .
\end{aligned}
$$

Let us now consider $j \leqq p$. From Lemma 3 below we have for $x_{j} \in \partial \Lambda$ and $y_{j}=\varepsilon^{-1} x_{j}$,

$$
\operatorname{div}_{x_{j}}^{\operatorname{cov}}\left(T_{x_{j}}\left(x_{j}\right) u\right)=T_{x_{j}}\left(x_{j}\right) \cdot \frac{\partial u}{\partial x_{j}}+\varepsilon^{-1} T_{x_{j}}\left(x_{j}\right) \cdot \frac{\partial u}{\partial y_{j}}+(d-1) u+(d-1) H\left(x_{j}\right)\left(x_{j} \cdot n_{j}\right) u .
$$

Integration by parts on $\partial \Lambda$ then gives

$$
\begin{aligned}
& -\sum_{j=2}^{p} \varepsilon^{-(n-1) d+p-3} \int_{(\partial \Lambda)^{p-1} \times \Lambda^{n-p}} d x_{2} \cdots d x_{n} x_{j} \cdot \frac{\partial u}{\partial y_{j}} \\
& =-\sum_{j=2}^{p} \varepsilon^{-(n-1) d+p-3} \int_{(\partial \Lambda)^{p-1} \times \Lambda^{n-p}} d x_{2} \cdots d x_{n}\left[T_{x_{j}}\left(x_{j}\right) \cdot \frac{\partial u}{\partial y_{j}}+\left(x_{j} \cdot n_{j}\right) n_{j} \cdot \frac{\partial u}{\partial y_{j}}\right]
\end{aligned}
$$




$$
\begin{aligned}
= & -\sum_{j=2}^{p} \varepsilon^{-(n-1) d+p-1} \int_{(\partial \Lambda)^{p-1} \times \Lambda^{p-1}} d x_{2} \cdots d x_{n}\left[\left(\varepsilon^{-2} x_{j} \cdot n_{j}\right) n_{j} \cdot \frac{\partial u}{\partial y_{j}}-\right. \\
& \left.-(d-1) H\left(x_{j}\right) u \varepsilon^{-1}\left(x_{j} \cdot n_{j}\right)-\varepsilon^{-1} T_{x_{j}}\left(x_{j}\right) \cdot \frac{\partial u}{\partial x_{j}}\right] \\
& +(p-1)(d-1) \varepsilon^{-(n-1) d+p-2} \int_{(\partial \Lambda)^{p-1} \times \Lambda^{n-p}} d x_{2} \cdots d x_{n} u .
\end{aligned}
$$

For later purposes, note that if $x_{j} \in \partial \Lambda$, then $\partial u / \partial x_{j}$ is tangent to $\partial \Lambda$ at $x_{j}$. Therefore

$$
\varepsilon^{-1} T_{x_{j}}\left(x_{j}\right) \cdot \frac{\partial u}{\partial x_{j}}=\varepsilon^{-1} x_{j} \cdot \frac{\partial u}{\partial x_{j}}
$$

Collecting all terms gives Lemma 2. Notice the cancellation of the singular terms (as $\varepsilon \rightarrow 0$ ) proportional to

$$
\varepsilon^{-(n-1) d+p-2} \int_{(\partial \Lambda)^{p-1} \times \Lambda^{n-p}} d x_{2} \ldots d x_{n} u\left(x_{2}, \ldots, x_{n} ; \varepsilon^{-1} x_{2}, \ldots, \varepsilon^{-1} x_{n}\right) . \quad \text { Q.E.D. }
$$

Lemma 3. Let $T_{x}(x)$ be the orthogonal projection of the vector $x \in \mathbb{R}^{d}$, defining a point $x \in \partial \Lambda$, onto the tangent plane at $x \in \partial \Lambda$. Then

$$
\operatorname{div}^{\operatorname{cov}} T(x)=d-1+(d-1) H(x) x \cdot n(x),
$$

where $H(x)$ is the mean curvature of $\partial \Lambda$ at $x$, defined with the inner normal $n(x)$ as follows: if $e_{1}, \ldots, e_{d-1}, n(x)$ is a moving frame at $x \in \partial \Lambda$ [3], then

$$
((d-1) H(x))=n(x) \cdot \sum_{l=1}^{d-1} d e_{l}\left(e_{l}\right)
$$

Proof. Let the 1 -forms $\sigma_{k}$ be defined by

$$
\sigma_{k}\left(e_{l}\right)=\delta_{k, l} \quad k=1, \ldots, d-1 ; l=1, \ldots, d-1
$$

so that

$$
\begin{aligned}
d x & =\sum_{1}^{d-1} e_{k} \sigma_{k}, \\
d e_{k} & =\sum_{l=1}^{d-1} d e_{k}\left(e_{l}\right) \sigma_{l}, \\
d \sigma_{k} & =\sum_{l=1}^{d-1} \sum_{l^{\prime}=1}^{d-1}\left(d e_{l}\left(e_{l^{\prime}}\right) \cdot e_{k}\right) \sigma_{l} \Lambda \sigma_{l}^{\prime} .
\end{aligned}
$$

The covariant divergence on $\partial \Lambda$ is defined, appropriately for integration by parts on $\partial \Lambda$, as

$$
\operatorname{div}^{\operatorname{cov}} T(x)=\sum_{k=1}^{d-1}(-)^{k-1} d\left\{\left(e_{k} \cdot T(x)\right) \sigma_{1} \wedge \cdots \wedge \check{\sigma}_{k} \wedge \cdots \wedge \sigma_{d-1}\right\} / \sigma_{1} \wedge \cdots \wedge \sigma_{d},
$$

where $T(x)$ could be replaced by any vector field in the tangent plane.

The proof of Lemma 3 is a straightforward computation, following the rules of 
exterior differentiation and using

$$
d e_{k}\left(e_{l}\right) \cdot e_{l}+d e_{l}\left(e_{l}\right) \cdot e_{k}=d\left(e_{k} \cdot e_{l}\right)\left(e_{l}\right)=0
$$

Proof of Theorem 1. By induction over $q$, consider first

$$
q=0, \quad k=0, \quad r=1 .
$$

In a neighborhood of the origin, the equation for $\partial \Lambda$ may be written

$$
x=\left(z_{\Lambda}(\mathbf{x}), \mathbf{x}\right),
$$

where $z_{\Lambda}(\mathbf{x})$ is a $C^{1}$ function of $\mathbf{x} \in \mathbb{R}^{d-1}$ and

$$
z_{\Lambda}(0)=0, \quad \frac{\partial}{\partial \mathbf{x}} z_{\Lambda}(0)=0
$$

The equation for $y \in \varepsilon^{-1} \partial \Lambda$ will then be

$$
y(\varepsilon, \mathbf{y})=\left(\varepsilon^{-1} z_{\Lambda}(\varepsilon \mathbf{y}), \mathbf{y}\right) .
$$

The uniform convergence of the integral defining $I_{n, p_{0}}(\varepsilon)$ means that for any $\eta>0$, there exists a ball $B \subset \mathbb{R}^{d}$ such that for all $\varepsilon \in\left[0, \varepsilon_{1}\right]$,

$$
\begin{aligned}
& \left|I_{n, p_{0}}(\varepsilon)-\int_{B^{n-1} \cap\left\{\left(\mathbb{R}^{d-1}\right)^{p_{0}-1} \times\left(\varepsilon^{-1} \Lambda\right)^{n-p_{0}}\right\}} d \mathbf{y}_{2} \cdots d \mathbf{y}_{p_{0}} d y_{p_{0}+1} \cdots d y_{n} \prod_{2} \prod_{0}^{p_{0}}\right| \frac{d y_{j}\left(\varepsilon \mathbf{y}_{j}\right)}{d \mathbf{y}_{j}} \mid \\
& \cdot u\left(y_{2}\left(\varepsilon, \mathbf{y}_{2}\right), \ldots, y_{p_{0}}\left(\varepsilon, \mathbf{y}_{p_{0}}\right), y_{p_{0}+1}, \ldots, y_{n}\right) \mid<\eta / 2,
\end{aligned}
$$

where $\varepsilon_{1}$ is such that $\varepsilon_{1} B$ is contained in the neighborhood of the origin as above.

The integrand is continuous in $\varepsilon \in\left[0, \varepsilon_{1}\right], \mathbf{y}_{2}, \ldots, \mathbf{y}_{p_{0}} \in \mathbb{R}^{d-1} \cap B, y_{p_{0}+1}, \ldots, y_{n} \in B$ and is therefore bounded by a constant. The characteristic function of $\left(\varepsilon^{-1} \Lambda\right)^{n-p_{0}}$ is also bounded. The dominated convergence theorem then implies that $I_{n, p_{0}}(\varepsilon)$ is continuous at $\varepsilon=0$.

We now proceed by induction over $q$. If Theorem 1 is valid up to $q-1$, it will be valid up to $q$ if we can apply it to $(d / d \varepsilon) I_{n, p_{0}}(\varepsilon)$. We only have to check the hypothesis for $v$ and $w_{j}$ in Lemma 2 .

The hypothesis

$$
\sum_{2}^{p} q_{j} \leqq q+|\alpha|+p-p_{0}
$$

enters as follows. Each derivative in $\varepsilon$ requires one moment (hence $q$ ), except when a derivative of $u$ with respect to $y_{j}$ is taken (hence $\left.|\alpha|\right)$, or when the dimension of the integration domain decreases by one (hence $p-p_{0}$ ). This concludes the proof of Theorem 1.

Theorem 2. Let $\Lambda \subset \mathbb{R}^{d}, \Lambda$ compact connected, and $\partial \Lambda$ of class $C^{r}$. Let $u_{n}\left(x_{1}, x_{2}, \ldots, x_{n}\right)$ be a $C^{k}$ function of $x_{1}, x_{2}, \ldots, x_{n} \in \mathbb{R}^{d}$, symmetric under permutations of $x_{1}, \ldots, x_{n}$, invariant under simultaneous translations of $x_{1}, \ldots, x_{n}$ Let $1 \leqq q \leqq$ 
$\operatorname{Min}(k+2, r)$ and suppose that all derivatives $D^{\alpha} u_{n}$ with respect to $x_{1}, x_{2}, \ldots, x_{n}$ of total order $0 \leqq|\alpha| \leqq \operatorname{Max}(0, q-2)$ satisfy $u_{n}\left(0, y_{2}, \ldots, y_{n}\right) \in L^{1}\left(\mathbb{R}^{(n-1) d}\right)$, and

$$
\int_{\partial \Lambda} d x_{1} \int_{\left(\varepsilon^{-1}\left(\partial \Lambda-x_{1}\right)\right)^{p-1} \times\left(\varepsilon^{-1}\left(\Lambda-x_{1}\right)\right)^{n-p}} d y_{2} \cdots d y_{n} \cdot \prod_{2}^{n}\left|y_{j}\right|^{q_{j}}\left|D^{\alpha} u_{n}\left(0, y_{2}, \ldots, y_{n}\right)\right|<c<\infty
$$

for all $\varepsilon \in\left[0, \varepsilon_{0}\right]$, for all $p$ with $1 \leqq p \leqq q$, for all $\left\{q_{2}, \ldots, q_{n}\right\}$ with

$$
\begin{aligned}
& q_{j} \geqq 0 \quad \forall j, \\
& q_{j} \leqq 1 \quad \text { if } \quad j>p, \\
& \sum_{2}^{n} q_{j} \leqq q+|\alpha|+p-1 .
\end{aligned}
$$

Suppose moreover that the above integrals are convergent, as any $\left|y_{j}\right| \rightarrow \infty$, uniformly in the other variables (particularly $\varepsilon$ and $x_{1}$ ). Then the function

is $C^{q}$ in $\varepsilon$ at $\varepsilon=0$. Moreover

$$
I_{n}(\varepsilon, \Lambda)=\varepsilon^{d} \int_{\left(\varepsilon^{-1} \Lambda\right)^{n}} d x_{1} \cdots d x_{n} u_{n}\left(x_{1}, \ldots, x_{n}\right)
$$

$$
\begin{aligned}
I_{n}(0, \Lambda)=|\Lambda| \int_{\left(\mathbb{R}^{d}\right)^{n-1}} d y_{2} \cdots d y_{n} u_{n}\left(0, y_{2}, \ldots, y_{n}\right) \equiv|\Lambda| C_{0}\left(u_{n}\right), \\
\frac{d}{d \varepsilon} I_{n}(0, \Lambda)=-\int_{\partial \Lambda} d x_{1} \int_{\left(\mathbb{R}_{x_{1}}^{d}\right)^{n-1}} d y_{2} \cdots d y_{n}\left(\sum_{2}^{n}\left(y_{j}-x_{1}\right) \cdot n_{1}\right) u_{n}\left(x_{1}, y_{2}, \ldots, y_{n}\right) \\
\equiv-|\partial \Lambda| C_{1}\left(u_{n}\right) \text { if } u_{n} \text { is rotation invariant, where } \\
\mathbb{R}_{x_{1}+}^{d}=\left\{x \in \mathbb{R}^{d}:\left(x-x_{1}\right) \cdot n_{1} \geqq 0\right\}, \\
\frac{d^{2}}{d \varepsilon^{2}} I_{n}(0, \Lambda)=\left\{\begin{array}{l}
0 \quad \text { if } n=2 \\
-\frac{1}{2}(n-1) \int_{\partial \Lambda} d x_{1} \int_{\mathbb{R}_{x_{1}}^{d-1}} d y_{2} \int_{\left(\mathbb{R}_{\left.x_{1}+\right)^{n-2}} d y_{3} \cdots d y_{n}\right.}
\end{array}\right. \\
\quad\left(\left(y_{2}-x_{1}\right) \cdot D n_{1}\left(y_{2}-x_{1}\right)\right)\left(\sum_{3}^{n}\left(y_{j}-x_{1}\right) \cdot n_{1}\right) u_{n}\left(x_{1}, y_{2}, \ldots, y_{n}\right) \\
\equiv+\frac{1}{2}(n-1)\left(\int_{\partial \Lambda} d x H(x)\right) C_{2}\left(u_{n}\right) \text { if } u_{n} \text { is rotation invariant, }
\end{aligned}
$$

where

$$
\mathbb{R}_{x_{1}}^{d-1}=\left\{x \in \mathbb{R}^{d}:\left(x-x_{1}\right) \cdot n_{1}=0\right\}
$$

and

$$
\begin{aligned}
& C_{2}\left(u_{n}\right)=\int_{\mathbb{R}_{x_{1}}^{d-1}} d y_{2}\left(y_{2}-x_{1}\right)^{2} \int_{\left(\mathbb{R}_{x_{1}}^{d}\right)^{n-2}} d y_{3} \cdots d y_{n}\left(\sum_{3}^{n}\left(y_{j}-x_{1}\right) \cdot n_{1}\right) u_{n}\left(x_{1}, y_{2} \cdots y_{n}\right), \\
& \frac{d^{3}}{d \varepsilon^{3}} I_{n}(0, \Lambda)=\frac{n-1}{4} \int_{\partial \Lambda} d x_{1} \int_{\mathbb{R}_{x_{1}}^{d-1}} d y_{2} \int_{\left(\mathbb{R}_{x_{1}}^{d}+\right)^{n-2}} d y_{3} \cdots d y_{n}\left(\left(y_{2}-x_{1}\right) \cdot D n_{1}\left(y_{2}-x_{1}\right)\right)^{2} .
\end{aligned}
$$




$$
\begin{aligned}
& \left\{\left(1+\left(\sum_{3}^{n}\left(y_{j}-x_{1}\right) \cdot n_{1}\right)\left(n_{1} \cdot \frac{\partial}{\partial y_{2}}\right)\right\} u_{n}\left(x_{1}, y_{2}, \ldots, y_{n}\right)\right. \\
& -\frac{(n-1)(n-2)}{4} \int_{\partial \Lambda} d x_{1} \int_{\left(\mathbb{R}_{x_{1}}^{d-1}\right)^{2}} d y_{2} d y_{3} \int_{\left(\mathbb{R}_{x_{1}}^{d}\right)^{n-3}} d y_{4} \cdots d y_{n}\left(\left(y_{2}-x_{1}\right) \cdot D n_{1}\left(y_{2}-x_{1}\right)\right) . \\
& \cdot\left(\left(y_{3}-x_{1}\right) \cdot D n_{1}\left(y_{3}-x_{1}\right)\right)\left(\sum_{4}^{n}\left(y_{j}-x_{1}\right) \cdot n_{1}\right) u_{n}\left(x_{1}, y_{2}, \ldots, y_{n}\right) \\
\equiv & \frac{(n-1)}{4}\left(\int_{\partial \Lambda} d x\left(H^{2}-\frac{2}{3} \frac{d-2}{d-1} K\right)\right) C_{3}\left(u_{n}\right) \text { if } n=2 \text { or } 3 \text { and } u_{n} \text { is rotation invariant, }
\end{aligned}
$$

where $K$ is the gaussian curvature.

Proof. $I_{n}(\varepsilon, \Lambda)$ can be put in the form

$$
I_{n}(\varepsilon, \Lambda)=\varepsilon^{-(n-1) d} \int_{\Lambda^{n}} d x_{1} \ldots d x_{n} u_{n}\left(0, \varepsilon^{-1}\left(x_{2}-x_{1}\right), \ldots, \varepsilon^{-1}\left(x_{n}-x_{1}\right)\right)
$$

We then compute, using integration by parts as in the proof of Lemma 2:

$$
\begin{aligned}
\frac{d}{d \varepsilon} I_{n}(\varepsilon, \Lambda)= & -\varepsilon^{-(n-1) d} \int_{\partial \Lambda \times \Lambda^{n-1}} d x_{1} \cdots d x_{n} \sum_{2}^{n}\left(\varepsilon^{-1}\left(x_{j}-x_{1}\right) \cdot n_{1}\right) \\
& \cdot u_{n}\left(0, \varepsilon^{-1}\left(x_{2}-x_{1}\right), \ldots, \varepsilon^{-1}\left(x_{n}-x_{1}\right)\right),
\end{aligned}
$$

where $n_{1}$ is the inner normal to $\partial \Lambda$ at $x_{1}$. Lemma 2 can now be applied to give

$$
\begin{aligned}
\frac{d^{2}}{d \varepsilon^{2}} I_{n}(\varepsilon, \Lambda)= & -(n-1) \varepsilon^{-(n-1) d+1} \int_{(\partial \Lambda)^{2} \times \Lambda^{n-2}} d x_{1}, \ldots, d x_{n}\left(\varepsilon^{-2}\left(x_{2}-x_{1}\right) \cdot n_{2}\right) \\
& \cdot\left(\sum_{2}^{n} \varepsilon^{-1}\left(x_{j}-x_{1}\right) \cdot n_{1}\right) u_{n}\left(0, \varepsilon^{-1}\left(x_{2}-x_{1}\right), \ldots, \varepsilon^{-1}\left(x_{n}-x_{1}\right)\right) .
\end{aligned}
$$

So far, it has not been necessary to compute any gradients with respect to variables on the boundary. This explains why the hypotheses in Theorem 2 are slightly milder than in Theorem 1.

We now give a general recursion formula:

Lemma 4. For $q \geqq 3$ we have

$$
\begin{aligned}
\frac{d^{q}}{d \varepsilon^{q}} I_{n}(\varepsilon, \Lambda)= & -\int_{\partial \Lambda} d x_{1} \sum_{p=2}^{\operatorname{Min}(q, n)} \frac{(n-1) !}{(n-p) !} \varepsilon^{-(n-1) d+p-1} \\
& \int_{(\partial \Lambda)^{p-1} \times \Lambda^{n-p}} d x_{2} \cdots d x_{n} F_{p, n}^{q}\left(x_{1} \cdots x_{p} ; \varepsilon^{-1}\left(x_{2}-x_{1}\right) \cdots \varepsilon^{-1}\left(x_{n}-x_{1}\right)\right)
\end{aligned}
$$

with

$$
\begin{aligned}
& F_{p, n}^{q}\left(x_{1} \cdots x_{p} ; y_{2} \cdots y_{n}\right) \\
& \quad=G_{p}^{q}\left(x_{1} ; x_{2} \cdots x_{p} ; y_{2} \cdots y_{p} ; \frac{\partial}{\partial y_{2}} \cdots \frac{\partial}{\partial y_{p}}\right)\left(\sum_{2}^{n} y_{j} \cdot n_{1}\right) u_{n}\left(0, y_{2} \cdots y_{n}\right) .
\end{aligned}
$$

The operators $G_{p}^{q}$ are defined recursively by

$$
G_{2}^{2}\left(x_{1} ; x_{2} ; y_{2} ; \frac{\partial}{\partial y_{2}}\right)=Q_{x_{1}}\left(x_{2}, y_{2}\right), \quad G_{1}^{2} \equiv 0
$$


where $Q_{x_{1}}(z, y)$ is defined as in Lemma 1 (where $x_{1}=0$ ) and

$$
\begin{aligned}
& G_{p}^{q+1}\left(x_{1} ; x_{2} \cdots x_{p} ; y_{2} \cdots y_{p} ; \frac{\partial}{\partial y_{2}} \cdots \frac{\partial}{\partial y_{p}}\right) \\
&= \sum_{j=2}^{p}\left\{-Q\left(x_{j}, y_{j}\right) n_{j} \cdot \frac{\partial}{\partial y_{j}}+(d-1) H\left(x_{j}\right) n_{j} \cdot y_{j}+\frac{\partial}{\partial x_{j}} \cdot y_{j}\right\} \\
& \cdot G_{p}^{q}(\cdot ; \cdots ; \cdots ; \cdots)+Q_{x_{1}}\left(x_{p}, y_{p}\right) G_{p-1}^{q}(\cdot \cdots ; \cdots ; \cdots),
\end{aligned}
$$

so that $G_{p}^{q}\left(x_{1} \cdots x_{p} ; y_{2} \cdots y_{p} ;\left(\partial / \partial y_{2}\right) \cdots\left(\partial / \partial y_{p}\right)\right)$ is a polynomial in $\left\{y_{j}\right\}_{j}$ and $\left\{n_{j} \cdot\left(\partial / \partial y_{j}\right)\right\}_{j}$ with coefficients continuous functions of $x_{1} \cdots x_{p} \in \partial \Lambda$. The order of $G_{p}^{q}$ (i.e. the degree in $\left\{\partial / \partial y_{j}\right\}_{j}$ ) is at most $q-2$. The total degree of $G_{p}^{q}$ in $\left\{y_{j}\right\}_{j}$ (counted positively) and $\left\{\partial / \partial y_{j}\right\}_{j}$ (counted negatively) is at most $q+p-1$.

Proof of Lemma 4 and Theorem 2. The proof is now straightforward, using Lemma 2 and Theorem 1.

Corollary 1. Let $\Lambda \subset \mathbb{R}^{d}, \Lambda$ compact connected and $\partial \Lambda$ of class $C^{r}$. Let

$$
u_{n}\left(x_{1}, x_{2}, \ldots, x_{n}\right)=\sum_{G \in g\left(x_{1}, \ldots, x_{n}\right)\left(x, x^{\prime}\right) \in G}\left(e^{-\beta \Phi\left(x, x^{\prime}\right)}-1\right),
$$

where $g\left(x_{1}, \ldots, x_{n}\right)$ is the set of graphs connecting $x_{1}, \ldots, x_{n}$ by two body unrepeated links $\left(x, x^{\prime}\right)$, and where the potential $\Phi\left(x, x^{\prime}\right)$ depends only on the distance $\left|x-x^{\prime}\right|$ and is stable and regular for moments up to $2 q-2$ :

$$
\forall n \in \mathbb{N}, \forall\left(x_{1}, \ldots, x_{n}\right) \in\left(\mathbb{R}^{d}\right)^{n}, \prod_{i<j}\left(1+\left|x_{i}-x_{j}\right|\right)^{2 q-2}\left|e^{-\beta \Phi\left(x_{i}, x_{j}\right)}-1\right|<e^{n \beta B_{q}}
$$

and

$$
\int_{\mathbb{R}^{d}} d x(1+|x|)^{2 q-2}\left|e^{-\beta \Phi(0, x)}-1\right|<C_{q}(\beta) .
$$

Suppose moreover that $e^{-\beta \Phi(0, x)}$ is $C^{k}$ in $x$ with $k=\operatorname{Max}(0, q-2)$ and

$$
\int_{\mathbb{R}^{d}} d x|x|^{q+\alpha}\left|\frac{d^{\alpha}}{d|x|^{\alpha}} e^{-\beta \Phi(0, x)}\right|<\infty
$$

for $1 \leqq \alpha \leqq q-2$.

Let the pressure $p\left(\varepsilon^{-1} \Lambda\right)$ in the volume $\varepsilon^{-1} \Lambda$ be defined by

$$
\beta\left|\varepsilon^{-1} \Lambda\right| p\left(\varepsilon^{-1} \Lambda\right)=\sum_{n=1}^{\infty} \frac{z^{n}}{n !} \int_{\left(\varepsilon^{-1} \Lambda\right)^{n}} d x_{1} \cdots d x_{n} u_{n}\left(x_{1}, \ldots, x_{n}\right)
$$

and the activity $z$ be such that

$$
z<e^{-\beta B_{q}}\left(C_{q}(\beta)\right)^{-1} .
$$

Then the pressure $p\left(\varepsilon^{-1} \Lambda\right)$ is $C^{q}$ in $\varepsilon$ at $\varepsilon=0$.

Proof. It is sufficient to prove

$$
\int_{\left(\varepsilon^{-1} \partial \Lambda\right)^{p-1} \times\left(\varepsilon^{-1} \Lambda\right)^{n-p}} d y_{2} \cdots d y_{n} \prod_{2}^{p}\left|y_{j}\right|^{q_{j}}\left|D^{\alpha} u_{n}\left(0, y_{2} \cdots y_{n}\right)\right|<n ! e^{n \beta B_{q}} C_{q}(\beta)^{n}(1+n)^{d_{q}}
$$


for some $d_{q}$ and all $n$, for all $\left\{q_{2}, \ldots, q_{p}\right\}$ and $\alpha$ and $\varepsilon$ as in Theorem 2 .

We replace $u_{n}$ by the Mayer expansion (1) and apply the derivatives. Leibniz's rule leads to $O\left(n^{|\alpha|}\right)$ choices of differentiated links. We now consider one such choice, so that at most $(q+1)$ links are differentiated.

We then note that every $y_{j}$ is connected to $y_{1}$ by a succession of links $y_{k}, y_{l}$ and replace $\left|y_{j}\right|$ in (2) by the bound

$$
\left|y_{j}\right| \leqq \prod\left(1+\left|y_{k}-y_{l}\right|\right)
$$

where the product runs along the path from $y_{j}$ to $y_{1}$.

We are now in a position to apply the Kirkwood Salsburg equation with the kernel

$$
\left(1+\left|y_{k}-y_{l}\right|\right)^{2 q-2}\left(e^{-\beta \Phi\left(y_{k}, y_{l}\right)}-1\right)
$$

for all links except the $q+1$ prescribed differentiated links. The bound (2) is then a standard result [2].

In Corollary 1 , it was assumed that the activity $z$ was small enough, but the result should be valid in the whole domain where the pressure is analytic in $z$, assuming bounds for the truncated correlation functions rather than for the Ursell functions. This requires a resummation of the expansion in $z^{n}$, and will be easier to formulate in terms of the average number of particles $\bar{N}\left(z, \varepsilon^{-1} \Lambda\right)$ or in terms of the average density

$$
\begin{aligned}
\bar{n}\left(z, \varepsilon^{-1} \Lambda\right) & =\left|\varepsilon^{-1} \Lambda\right|^{-1} \bar{N}\left(z, \varepsilon^{-1} \Lambda\right)=\beta z \frac{\partial}{\partial z} p\left(z, \varepsilon^{-1} \Lambda\right) \\
& =\sum_{n=1}^{\infty} \frac{z^{n}}{(n-1) !}|\Lambda|^{-1} I_{n}(\varepsilon, \Lambda) \quad \text { if } z \text { small. }
\end{aligned}
$$

Let $\rho_{m}^{\Lambda}\left(x_{1} \cdots x_{m}\right)$ be the $m$-point truncated function at activity $z$ in volume $\Lambda$. For small $z$, we have

$$
\rho_{m}^{\Lambda}\left(x_{1} \cdots x_{m}\right)=z^{m} \sum_{n=0}^{\infty} \frac{z^{n}}{n !} \int_{\Lambda^{n}} d y_{1} \cdots d y_{n} u_{m+n}\left(x_{1} \cdots x_{m} y_{1} \cdots y_{n}\right) .
$$

When $x_{1} \in \partial \Lambda$, let $\varepsilon^{-1}\left(\Lambda-x_{1}\right)$ and $\varepsilon^{-1}\left(\partial \Lambda-x_{1}\right)$ be the transforms of $\Lambda$ and $\partial \Lambda$ under a scaling of amplitude $\varepsilon^{-1}$ and center $x_{1}$. We can now formulate Corollary 2:

Corollary 2. Under the hypotheses of Corollary 1, the average density $\bar{n}\left(z, \varepsilon^{-1} \Lambda\right)$ is $C^{q}$ in $\varepsilon$ at $\varepsilon=0$ and

$$
\begin{aligned}
\frac{d^{q}}{d \varepsilon^{q}} \bar{n}\left(z, \varepsilon^{-1} \Lambda\right)= & -|\Lambda|^{-1} \int_{\partial \Lambda} d x_{1} \sum_{p=2_{1}\left(\varepsilon^{-1}\right.}^{q} \int_{\left.\left(\partial \Lambda-x_{1}\right)\right)^{p-1}} d y_{2} \cdots d y_{p} \\
& \cdot G_{p}^{q}\left(x_{1} ; x_{1}+\varepsilon y_{2}, \ldots, x_{p}+\varepsilon y_{p} ; y_{2} \cdots y_{p} ; \frac{\partial}{\partial y_{2}} \cdots \frac{\partial}{\partial y_{p}}\right) . \\
& \cdot\left\{\left(\sum_{2}^{p} y_{j} \cdot n_{1}\right) \rho_{p}^{\varepsilon^{-1}\left(\Lambda-x_{1}\right)}\left(x_{1} y_{2} \cdots y_{p}\right)\right. \\
& \left.+\int_{\varepsilon^{-1}\left(\Lambda-x_{1}\right)} d y_{p+1}\left(y_{p+1} \cdot n_{1}\right) \rho_{p+1}^{\varepsilon-1\left(\Lambda-x_{1}\right)}\left(x_{1} y_{2} \cdots y_{p+1}\right)\right\},
\end{aligned}
$$

where $G_{p}^{q}$ is defined in Lemma 4. 
Suppose now that for all $\varepsilon \in] 0, \varepsilon_{0}\left[, \bar{n}\left(z, \varepsilon^{-1} \Lambda\right)\right.$ and its $q$ first derivatives with respect to $\varepsilon$ have an analytic continuation to a domain $D$ containing the origin and are bounded uniformly in $\varepsilon \in] 0, \varepsilon_{0}[$.

Then $\bar{n}\left(z, \varepsilon^{-1} \Lambda\right)$ and $p\left(z, \varepsilon^{-1} \Lambda\right)$ are $C^{q}$ in $\varepsilon$ at $\varepsilon=0$ for all $z \in D$.

Proof. The first part of the corollary is just a computation. The second part follows from Vitali's theorem. Note that the formula given in the first part is meant to be used for bounding the derivatives of $\bar{n}\left(z, \varepsilon^{-1} \Lambda\right)$ when using the second part of the corollary.

Acknowledgements. It is a pleasure to acknowledge useful discussions with our colleagues at Centre de Physique Théorique and also Centre de Mathématiques, Ecole Polytechnique. F.D. thanks the theoretical physics group at Université de Lausanne for the opportunity to give a seminar; the present paper was improved (we hope so) after this seminar, and also after the remarks of the referee which we gratefully acknowledge.

\section{References}

1. Pogosian, S.: Asymptotic expansion of the logarithm of the partition function. Commun. Math. Phys. 95, 227-245 (1984), and references therein

2. Ruelle, D.: Statistical mechanics, rigorous results. New York, Amsterdam: Benjamin 1969

3. Stoker, J. J.: Differential geometry. New York: Wiley 1969

Communicated by J. Fröhlich

Received December 16, 1985; in revised form June 23, 1986 